FORMATION Formation emploi

Revue française de sciences sociales

144 | Octobre-Décembre

Le décrochage scolaire à l'aune des inégalités

territoriales: nouvelles perspectives?

\title{
Mésalliance éducative : l'exemple d'un partenariat territorialisé de lutte contre le décrochage scolaire
}

Educational misalliance: the example of a territorialized partnership to fight school dropout

Fehlendes Bildungsbündnis? Beispiel einer territorialen Partnerschaft zur

Bekämpfung des vorzeitigen Schulabgangs

Mala alianza educativa : el ejemplo de una asociación territorializada de lucha

contra el abandono escolar

Laure Minassian

\section{OpenEdition}

Journals

Édition électronique

URL : http://journals.openedition.org/formationemploi/6522

DOI : $10.4000 /$ formationemploi.6522

ISSN : 2107-0946

Éditeur

La Documentation française

Édition imprimée

Date de publication : 31 décembre 2018

Pagination : 73-93

ISSN : 0759-6340

Référence électronique

Laure Minassian, « Mésalliance éducative : l'exemple d'un partenariat territorialisé de lutte contre le décrochage scolaire », Formation emploi [En ligne], 144 | Octobre-Décembre, mis en ligne le 31 décembre 2019, consulté le 06 janvier 2021. URL : http://journals.openedition.org/formationemploi/ 6522 ; DOI : https://doi.org/10.4000/formationemploi.6522 


\title{
Mésalliance éducative : l'exemple d'un partenariat territorialisé de lutte contre le décrochage scolaire
}

\begin{abstract}
Laure Minassian
Docteure en sciences de l'éducation et de la formation, chercheure en sociologie de l'éducation au laboratoire CIRCEFT-ESCol (Centre Interdisciplinaire de Recherche, Culture, Éducation, Formation - Éducation et Scolarisation) de Paris 8. Ingénieure de recherches, cheffe de projet Formation-Enseignement supérieur au sein de I'I-SITE NEXT (Nantes Excellence Trajectory), qui associe l'École centrale-Nantes, I'Université de Nantes, le CHU (Centre hospitalier universitaire) de Nantes et I'INSERM (Institut national de la santé et de la

recherche médicale)
\end{abstract}

Résumé

Mésalliance éducative : l'exemple d'un partenariat territorialisé de lutte contre le décrochage scolaire

Peu de travaux de recherche se sont intéressés de façon longitudinale aux dispositifs de lutte contre le décrochage scolaire, depuis leur conception politique jusqu'à leur déploiement opérationnel. C'est ce que propose cette contribution par le biais d'une étude de cas où s'articulent une Région, une association et des lycées professionnels, au sein d'un dispositif appelé "alliances éducatives ". L'analyse vise à mettre au jour comment les interactions entre une politique, l'institution scolaire, les acteurs associatifs et les élèves génèrent des effets adverses à ceux souhaités.

Mots clés : abandon des études, mesure jeune, approche locale, région, lycée professionnel, organisation du travail, projet d'établissement

Abstract

Educational misalliance: the example of a territorialized partnership to fight school dropout

Few research have focused on longitudinal studies to investigate new educational meso-systems to fight early school leaving. This contribution aims to focus in from their political conception until their operational deployment, through a case study of a Region, an association and three vocational high schools, within a device called "educational alliances". The analysis aims to reveal how the interactions between a policy, the school institution, the associative actors and the students generate effects adversaries to those desired. 
Keywords: keywords: drop out, youth employment scheme, local approach, region, vocational upper secondary school, work organisation, school work plan

Journal of Economic Literature: I 28 ; R 58

Traduction : Auteure.

\section{Introduction}

À la suite de la massification progressive de l'enseignement secondaire, la volonté des pouvoirs publics de lutter contre l'échec scolaire et ses conséquences a conduit à emprunter successivement deux voies : la standardisation du système scolaire jusqu'au milieu des années 1980 (Bourquelot ; Derouet, 1994) ; suivi du déclin du paradigme Républicain ${ }^{1}$, au profit de la territorialisation des politiques éducatives.

Pourtant l'adaptation des politiques nationales aux spécificités locales ne donne pas toujours lieu aux effets escomptés. Les logiques d'acteurs ou de groupe d'acteurs tout à la fois interdépendantes et concurrentielles constituent parfois un obstacle (Dutercq \& al., 2015 ; Ben Ayed, 2009), bien qu'au cours du temps des modalités d'actions ont pu émerger, et donner lieu à des cultures communes professionnelles (Rey, 2013). Sur la base d'un matériau empirique, cette contribution vise à questionner quelques effets de telles logiques dans de nouveaux dispositifs territorialisées, au regard de thématiques plus générales ayant trait à la question des inégalités et des ségrégations sociales et scolaires (Broccolochi et al., 2007).

Pour ce faire, on s'intéresse à ce qui se joue en bas, en donnant à voir des processus territoriaux de négociation et de gestion de l'action publique. Le cas étudié articule une Région, une association à vocation d'éducation populaire (l'Association Fondation pour les Étudiants de la Ville, AFEV), et trois lycées professionnels caractérisés par des taux de décrochage scolaire importants. La Région dispose d'une certaine marge de manœuvre motivée par de nouvelles préconisations nationales et européennes de lutte contre le décrochage scolaire, qui, comme le montrent les travaux de Bruno et al. (2013), accordent une attention plus large aux contours de ce phénomène et encouragent à agir au plus près de l'expérience des élèves. Il en va ainsi du rapport de la Commission européenne (2005) dans lequel trois types de mesures sont mentionnées : l'aide financière à la scolarité pour les élèves de milieux sociaux défavorisés ; des mesures compensatrices et préventives au sein des cursus scolaires dans lesquelles s'inscrit l'individualisation des parcours; le soutien scolaire par des modules d'orientation et de réorientation, de tutorat (Bruno \& al., 2017). Soulignons d'ailleurs les

1. La tradition française centralisatrice qui s’exprime par les politiques éducatives jusque dans les années 1980 s'inspire d'une pensée universaliste et méritocratique. La notion d'égalité, héritée de la Révolution française (égalité en droit, instruction gratuite et obligatoire) était censée garantir le développement des " talents " de chacun. L'échec de cette idéal politique a conduit à de nouvelles formes d'articulation du national et du local. 
effets positifs de ces politiques ; elles ont participé d'une lente décroissance des nondiplômés, sans pour autant annuler ce phénomène (Bernard, 2011).

Des études récentes interrogent les effets de l'accroissement de ces dispositifs (Millet \& Thin, 2017 ; Barrère, 2013 ; Kakpo \& Netter, 2013 ; Minassian, 2017 ; Pirone \& Rayou, 2012), mais elles prennent peu ou pas en compte le développement des interactions et des partenariats qui les régissent : entre des acteurs de collectivités (avec leur personnel administratif), d'établissements scolaires (chefs d'établissement, mais aussi CPE (Conseillers Principaux d'Éducation)), et de groupes d'intérêts impliqués (associations, parents, etc.). L'originalité de cette contribution est sa focalisation sur ces interactions au travers de l'étude de cas d'un dispositif appelé "alliances éducatives » inspiré du plan ministériel "Tous mobilisés pour vaincre le décrochage scolaire ", présenté le 21 novembre 2014 par Manuel Valls, alors Premier ministre et Najat VallaudBelkacem, ministre de l'Éducation nationale, de l'Enseignement supérieur et de la Recherche sur cette période 2 .

Le dispositif " alliances éducatives " vise à ouvrir l'école à, à faire alliance avec, des institutions qui en sont traditionnellement éloignées (familles, associations, protection judiciaire de la jeunesse, etc.) (Armand \& al., 2013). Comme d'autres dispositifs, il est utilisé pour remédier aux dysfonctionnements de l'école et constitue " un faire faire " par autrui ce que l'école ne peut plus assurer ordinairement (Barrère, op. cit., p. 98). En visant un partage horizontal de la prise en charge de la difficulté des élèves, il s'agit de mobiliser non plus une équipe éducative (interne à l'école), mais une communauté élargie qui s'étend au-delà de l'institution scolaire dans une "injonction à la proximité" (Guiliani, Payet, 2014) à différents niveaux : "à un niveau micro, en partenariat "jeune-famille-école ", à un niveau méso en sétendant à d'autres acteurs des sphères sociale, judiciaire ou du monde de la santé. Enfin, à un niveau macro englobant les niveaux micro et méso, des dispositifs communautaires peuvent mettre en ouvre de larges alliances au sein de régions ou d'États (...)»(Blaya, 2011, p. 241).

L'approche proposée est ethnographique et relationnelle. Elle conjugue deux cadres théoriques : la théorie de l'ordre négocié (Strauss, 1978) et l'analyse du discours, dans la continuité des travaux de Boutet \& Maingueneau (2005). La théorie de l'ordre négocié permet d'envisager la mise en place d'un partenariat comme un monde social spécifique avec ses enjeux propres. L'étude de la répartition des rôles, de la manière dont bougent les lignes et les statuts, rend visible l'asymétrie entre les différents acteurs et explique, pour une part, les contextes d'action. L'analyse du discours donne à voir, au travers de productions d'énoncés, un contexte social dans et par lequel se coconstruisent des inégalités.

2. Le plan "Tous mobilisés pour vaincre le décrochage scolaire " s'articule autour de trois axes : la mobilisation de tous, le choix de la prévention, une nouvelle chance pour se qualifier. 
La première partie de la contribution détaille les critères politiques qui président à la mise en œuvre du dispositif ; la seconde analyse les contextes d'action ainsi générés au regard des contextes de gouvernance de trois lycées professionnels ; enfin, la troisième partie rend compte des actions rapportées par les animateurs enquêtés. En travaillant à des niveaux multiples, le propos rend visible l'écart entre la volonté politique d'origine et l'appropriation du projet par les acteurs locaux.

\section{Un engagement politique variable selon la place des acteurs dans l'espace social}

Rendre compte d'une expérimentation d'alliances éducatives et des effets produits nécessite de lever le voile sur différents niveaux dont relève un partenariat. Les tentatives des acteurs pour définir, en amont, la situation, les stratégies mises en œuvre pour exercer ou conserver le contrôle ou s'en accommoder au fil du temps, dépendent de la position de chacun dans l'espace social et constituent une composante même du dispositif étudié (Strauss, op. cit.)

Pour objectiver ces éléments, le propos emprunte à la théorie de l'ordre négocié. Elle vise moins à prendre en compte les organisations comme des déjà-là des systèmes figés et rigides, que comme des systèmes reconstruits dans et par l'action : "les organisations ne sont pas des formations structurées par des règles normatives univoques, les actions qui s'y déroulent ne sont pas déterminées par la simple application de prescriptions ou de règles de conduites libres de toute intervention de la personne de l'acteur " (Baszanger, in Strauss, 1992, p. 45). Dans cette perspective, les manières de redéfinir le décrochage, les conflits, les changements, les consensus qui en découlent sont d'importance pour donner à voir le cadre général dans lequel le dispositif se déploie.

Ce cadre d'analyse est conjugué avec l'approche cognitive de l'analyse du discours, dans la continuité des travaux sociolinguistiques de Boutet \& Maingueneau (op. cit.). Celle-ci permet, d'une part, de rendre compte du sens que les acteurs confèrent à leurs actions (Schmoll, 1996); d'autre part, d'expliquer, en partie, des manières de redéfinir des rôles, des règles. Plus précisément, les usages langagiers et ce qu'ils fabriquent, ce pourquoi ils sont mobilisés, fournissent l'occasion de saisir des indices de sens au sujet de modes d'interprétation socialement situés, selon les lieux, les individus en présence. 


\section{Encadré 1. Trianguler les données : le contexte social des trois lycées professionnels retenus}

Le dispositif d'« alliances éducatives » constitutives du propos, a fait l'objet d'une enquête ethnographique conduite sur deux années $(*)$. Elle a permis de suivre l'ensemble des acteurs impliqués : commanditaires, chefs d'établissement, CPE (conseiller principal d'éducation), cadres de l'association, animateurs. Les données de l'enquête comprennent des prises de notes effectuées lors de réunions organisées par la Région, des entretiens longs avec les animateurs (deux séries de 12 entretiens d'une à deux heures chacun), mais aussi CPE, élèves, chefs d'établissement, des séries d'observations dans les établissements (auprès des bureaux de la vie scolaire, dans la cour, auprès des animateurs, pendant la pause méridienne, lors de séances d'enseignement, autour du pôle scolarité où se rendent les élèves exclus de cours).

L'ensemble de ces corpus mis en relation permet de retracer une perspective d'ensemble des effets produits. La méthode de traitement des données est « contrastive » II agit d'analyser les interviews, prises de parole dans l'enceinte scolaire, autour d'une table de réunion au rectorat, etc., pour donner à voir quelques représentations à l'oeuvre à partir de la façon dont les locuteurs utilisent le langage pour parler d'eux-mêmes et du dispositif.

Faisant l'objet d'une autre publication à venir, les observations des élèves et des enseignants en établissement ne seront pas présentées ici. En outre, I'attention accordée au dispositif plus qu'aux trajectoires des acteurs constitue l'une des limites de cette étude.

L'enquête s'étend au-delà des données ethnographiques, pour s'attacher aussi au contexte macro-socio-géographique des territoires dans lesquels les lycées professionnels observés sont implantés.

Le graphique 1 permet de décrire le profil des communes où sont situés les lycées sous l'angle de la structure des emplois (taux d'employés, ouvriers, retraités), de la composition des familles (familles monoparentales), de l'activité et de l'emploi (taux de chômage), du logement (taux de résidences HLM - Habitation à loyer modéré), de la scolarisation (taux de nondiplômés, non scolarisés de plus de 15 ans), du taux de pauvreté.

$\left.{ }^{*}\right)$ : Cette recherche a reçu le soutien de l'Agence nationale de la recherche - convention ANR-14-CE30-0009. 


\section{Graphique 1. Profil des communes de localisation des lycées. Comparatif avec France entière.}

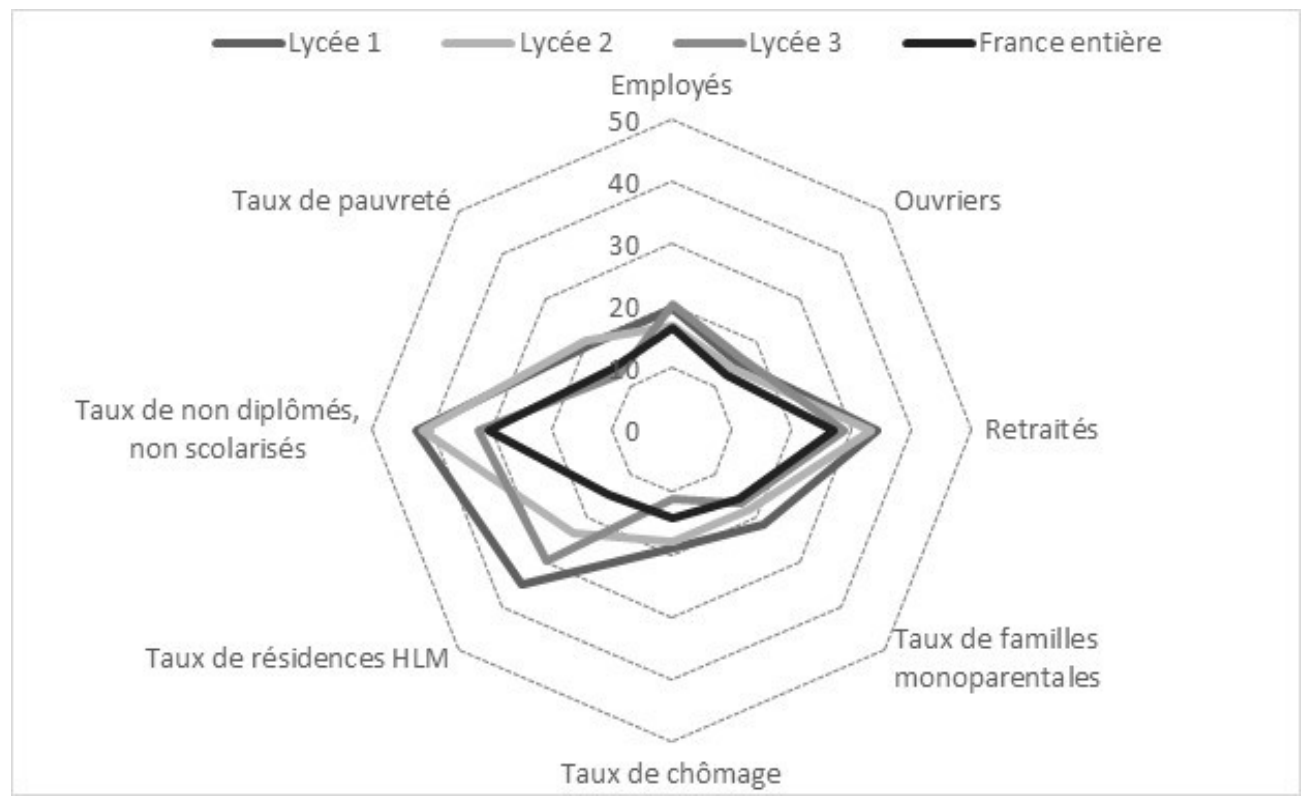

Source : Insee, RP2015 exploitation principale, géographie au 01/01/2017, traitement auteure.

Les communes de localisation des lycées 1 et 2 ont un profil social proche, malgré leurs spécificités. Elles sont globalement en difficulté, si l'on s'en tient au taux de pauvreté (19\% pour la commune du lycée $1 ; 20.3$ \% pour celle du lycée 2 , INSEE, 2018) et de chômage (18 \% pour la commune du lycée $1,19 \%$ pour celle du lycée 2 , id.). La commune sur laquelle est situé le lycée 1 est une ancienne cité ouvrière dans laquelle l'usine principale a fermé ses portes dans les années 1980. Sa population a fluctué, entre désertification au moment des vagues de licenciements et léger repeuplement depuis les années 2000.

La commune de localisation du lycée 3 possède un profil social et culturel en demi-teinte. Le taux de pauvreté est inférieur aux deux autres communes ( $12 \%$ pour la commune, contre $14 \%$ France entière, INSEE, 2018), ainsi que le taux de chômage ( $15.3 \%$ pour la commune contre $9 \%$ France entière, INSEE, 2018), du fait, entre autres, de la proximité avec une ville de taille moyenne.

\subsection{Dans sa lutte contre le décrochage, la région cible des lycées professionnels...}

Dans les pays de l'OCDE (Organisation de coopération et de développement économiques), la tendance générale à mettre en place un système éducatif moins coûteux et plus souple (Pont \& al., 2008) a en partie conduit au développement de la contractualisation et à la mise en place d'objectifs de performance mesurables (Pollitt \& Bouckaert, 2011 ; 
Bezes, 2009 ; Hood, 1991). Dans le cas précis, la signature du Contrat État-Région 20152020, en particulier le volet "Emploi-Orientation-Formation professionnelle " relève de ce double principe (L.2014-288 du 5 mars 2014).

Concernant la lutte contre le décrochage, la contrainte exercée par les indicateurs de performance, en particulier le taux de décrochage scolaire, a conduit les opérateurs de politiques publiques à adopter des pratiques gestionnaires qui s'inscrivent dans une logique objectifs/moyens/résultats (Attarça \& Chomienne, 2013). À la Région, la manière dont ces indicateurs sont perçus par les administratifs, conduit à cibler les lycées professionnels où les décrocheurs sont en forte concentration, afin de réduire globalement leur nombre. En partie pour euphémiser ce calcul stratégique, la désignation de ces établissements par la Région a été justifiée au regard de critères sociogéographiques - transformant ces derniers en établissements ruraux (en réalité deux établissements sur trois le sont) et faisant des territoires des lieux où se conjugueraient des difficultés sociales notamment liées, à la mobilité, au chômage, à une offre de loisirs et d'infrastructures faible.

Structuré verticalement, le groupe des cadres impliqués relève de services proches, mais de statuts fort différents : une chargée de mission "Prospective, Métiers, Carte des formations", un chef de projet "Conduite de projets, Prospective Métiers-Orientation-Qualification Économie/Éducation-Carte des Formations", le directeur du service "Formation tout au long de la vie " ${ }^{3}$.

Cette hiérarchie des champs de compétences associée aux différences de genre, donne lieu à une division sociale du travail. La position haute est celle du directeur de service, la position basse celle de la chargée de mission. Cette distinction explique en partie la constante implication de cette dernière. En effet, elle vient présenter le projet, se déplace au siège de l'association, à l'université, au rectorat, au contraire de ses deux collègues, dont l'un minimise sa parole et son investissement (le chef de projet qui se trouve statutairement dans une position d'entre-deux), au contraire du second (le Directeur du service "Formation tout au long de la vie ").

La présence du chercheur lors des réunions organisées par la Région permet d'objectiver, outre une régulation interindividuelle à l'interne, une politique adoptée par l'institution plutôt descendante envers les établissements. Le "privilège du petit nombre " (Wieser, 1901, in Weber, 2013, p. 70) confère un rapport de force formellement en faveur des trois cadres administratifs. Ils peuvent en effet se consulter dans un délai restreint (leurs bureaux sont situés dans le même bâtiment et sur le même étage), s'accorder sur la manière de définir l'action, redéfinir les territoires d'intervention et imposer en apparence une politique aux chefs d'établissements désignés, nous y reviendrons.

L'invitation aux réunions de copilotage concerne deux fois plus souvent les représentants de l'AFEV que les chefs d'établissements. Concrètement, l'AFEV, les chefs d'établissement et

3. On ignore les raisons de ce choix organisationnel et l'avis de leur hiérarchie. 
le rectorat sont réunis une fois en début d'année et une fois en fin d'année. Entre ces deux moments, l'AFEV expose ses actions une fois par trimestre auprès des cadres de la Région. À l'occasion de ces rencontres trimestrielles, le directeur de service et son équipe exposent leurs représentations des acteurs de l'école et de leurs modes d'intervention : le décrochage scolaire est alors imputé presque exclusivement à un "manque de compétences de la part de l'Éducation nationale ", en deuxième lieu "à la famille " (Directeur de service). L'école est alors décrite comme "cloisonnée ", "formelle " et peu ouverte "aux savoirs de l'expérience", à "l'initiative ", à la notion de "talents" (Directeur de service). Ce discours organise des rapports sociaux et laisse une plus ou moins grande marge de manœuvre aux acteurs impliqués. L'AFEV en est la principale bénéficiaire.

\section{2.... au service du développement de l'association}

La désignation d'une association intersectorielle qui traverse notamment les politiques éducatives, de la jeunesse, du logement et de la ville, pour assurer des missions en milieu rural (suivant la requalification des établissements opérée), a pu séduire les cadres de la Région. En affinité avec les attentes du Directeur de service, lors des réunions, l'association véhicule une certaine représentation de la jeunesse idéalisée, et évoque peu souvent l'école.

\subsubsection{Une représentation homogène des jeunes...}

Cette représentation est visible sur le site web de l'association, au travers de techniques communicationnelles inspirées du monde marchand, amplifiées par les contenus photographiques ou vidéos où est mise en scène une jeunesse belle, dynamique, ouverte, curieuse. Les jeunes y apparaissent impliqués dans des projets culturels, toujours enthousiastes, "volontaires» (un terme qui emporte plus de 40 occurrences sur le site ${ }^{4}$ ). Certes, la concurrence dans ce secteur oblige les associations à se démarquer les unes des autres en affichant des valeurs et objectifs précis pour emporter l'adhésion de son public (Carion, 2010), c'està-dire à mettre en ouvre une stratégie dite de "social marketing " (Kotler, Zaltman ; 1971). Elle présente ici néanmoins deux faces d'un même problème : l'homogénéité d'une catégorie qui n'existe pas ("La jeunesse n'est qu'un mot», Bourdieu, 1978), et la projection d'une norme de ce que devrait être la jeunesse :

"On a l'impression d'une homogénéité des goûts des adolescents que parce que l'on ne veut pas apercevoir les divisions réelles : parce que la culture adolescente ne constitue jamais le tout de la culture des adolescents, leurs pratiques et leurs préférences échappent au moins partiellement aux modèles qu'elle propose, de sorte qu'elles dépendent au moins autant de la sous-culture de classe à laquelle ils participent" (Chamboredon, 1966).

4. Le logiciel Tagcrowd génère des «nuages de mots" à partir d'un texte source. Les nuages donnent plus d'importance aux mots les plus fréquents. Concernant le site de l'AFEV, aux côtés des termes "volontaires", on trouve aussi les occurrences «solidaires» et " engagement» de façon fréquente (plus de 40 occurences). La proximité sémantique entre ces termes mériterait d'ailleurs un approfondissement. 
D'ailleurs, une analyse plus approfondie du site fournit des indices de cette jeunesse idéalisée. Elle est ainsi plutôt urbanisée et de classe moyenne, et forme la trame de l'ensemble des contenus du site Web, sous l'opposition de deux espaces distincts : la ville et la campagne 5 . Eloignée de ce qui fait la spécificité des milieux ruraux, l'association s'est en effet plutôt construite dans l'éducation populaire urbaine, et s'est développée surtout autour d'une dynamique de quartiers. L'attention accordée au décrochage scolaire en milieu rural doit beaucoup à l'émergence, d'une part, d'une politique instituant le service civique (L.2010-241 du 10 mars 2010, Arrêté du 19 mai 2015) ; d'autre part, à la proposition faite par la Région d'une mission en établissement venant conforter sa volonté d'accroître et d'élargir son domaine d'expertise. Là encore, le site web de l'association nous renseigne sur cette volonté de développement : sur 19 offres d'emploi, 15 portent l'intitulé «chargé de développement local " ${ }^{6}$.

Pour convaincre de leurs compétences de conversion d'une animation hors école à des interventions dans l'école d'une part, et d'une expertise des quartiers populaires transposable aux milieux ruraux d'autre part, les représentants de l'association ont usé d'une phrase qui deviendra par la suite un credo repris par les cadres de la Région « d'une jeunesse qui va à la rencontre d'une autre jeunesse de façon spontanée ». En cohérence avec cet objectif vague, le programme en direction des élèves vise officiellement moins l'amélioration de leurs performances scolaires que la lutte contre l'absentéisme grâce à la fréquentation de jeunes en service civique qui, lors d'animations ponctuelles, ont pour objectif de redonner sens à l'école, sur deux demi-journées par semaine.

\subsection{2 ... qui participe d'un classement}

Pour l'AFEV, la négociation avec la Région occupe une place centrale au début comme au cours de la mise en œuvre du dispositif. Elle lui permet, tout d'abord, de revendiquer une position sociale en s'affirmant comme étant du côté de la jeunesse, se distinguant ainsi de l'école, plutôt centrée sur les savoirs. Elle l'utilise, ensuite, pour réorganiser les rapports sociaux, en précisant, lors d'une réunion à huis clos, et à la suite d'un bilan en demi-teinte, que "l'accompagnement ne marchera pas avec un élève en situation de grand décrochage " (Responsable locale de l'association accompagnée de la Responsable nationale), preuve que ses catégories ne l'aident guère. En effet, cette réorganisation de la négociation est tout à la fois la conséquence de l'occultation de l'hétérogénéité des jeunes et l'effet du maintien de logiques pratiques par le renvoi de l'inadéquation de la démarche du côté des élèves. Cette stratégie permet à l'association de poursuivre son développement. En cherchant un

5. Loin d'être insignifiante, l'une des vidéos (comme beaucoup d'autres) publiées pour promouvoir une ville de province focalise les 30 premières secondes, sur une durée de moins de 5 minutes, sur le départ en train des deux jeunes femmes " reporters ", depuis la gare Saint Lazare à Paris. Tout au long du trajet en train, des oppositions rural/urbain sont visibles (la campagne grise et pluvieuse, au contraire de l'ambiance joyeuse dans le train ; le car serpentant la campagne comme image d'Epinal vue du train, etc.).

6. Site consulté en novembre 2017. 
client idéal (dans le cas précis, des élèves de bonne volonté scolaire), l'association, classe et exclut, alors même qu'elle dénonçait ce comportement au début de l'enquête.

Par son statut juridique, l'association est libre de ses procédures de recrutement. Elle choisit ses animateurs parmi de nombreux candidats du fait d'un contexte d'emploi tendu selon des qualités personnelles. Dans les faits, l'analyse montre que les animateurs sont plutôt issus des classes moyennes (leurs parents sont plutôt issus de l'enseignement ou du travail social), ont dans leur très grande majorité rencontré des difficultés dans l'enseignement supérieur, disent être à un moment charnière et disent simultanément "vouloir être utile ", "être dans l'écoute ", "favoriser les échanges, les relations humaines ». Mais là au-delà de cette bonne volonté, contrairement à une volonté de professionnalisation dont elle s'estime porteuse, l'association ne délivrera pas ou peu de formation, si ce n'est celle qui consiste à former les animateurs à recruter d'autres volontaires dans le cadre de la mise en place de bénévolats cette fois, et valorisera auprès des jeunes en service civique la figure de l'autodidacte.

\section{Le rôle décisif des chefs d'établissement dans le partenariat}

Le contexte général de l'injonction à accueillir un nouveau dispositif se double d'un second contexte local. En s'intéressant à l'organisation des établissements, aux discours des personnels, à ce qu'ils disent faire, des indices se dégagent. Si dans les réunions préparatoires, chacun peut avoir le sentiment que la Région dirige, les établissements restent des structures relativement autonomes et s'accommodent plus ou moins de l'imposition qui leur est faite. Les actions à l'interne déterminent, en partie, une redéfinition du partenariat et rendent visibles l'asymétrie entre les établissements et l'association. Les paragraphes suivants donnent à voir les contours de cultures éducatives d'établissements.

\subsection{Le lycée 1 : une centration autour de la vie scolaire}

Dans le lycée 1, au début de l'enquête (janvier 2015), le chef d'établissement est remplacé par un adjoint de collège suite à de grandes difficultés internes identifiées par le Rectorat. Celles-ci apparaissent assez nettement dans les propos d'un des CPE interrogé dans le cadre de l'enquête : "Ici [au sujet de l'établissement] ce n'est pas le Bronx », ou encore dans ceux du chef d'établissement : "Ici ce n'est pas Chicago ". La négation d'une mauvaise réputation, qui reste à la fois une occultation et une désignation de la violence (ce n'est pas le Bronx, Chicago), par la référence à des villes américaines connues pour leur violence urbaine, tandis qu'on se situe dans le bocage, reste une analogie intéressante à double titre. D’une part, elle lève le voile sur une opinion générale et la réputation que porte l'établissement. D'autre part, elle nous apprend qu'il existe d'autres éléments fins, étrangers aux 
caractéristiques territoriales, similaires à certains discours au sein d'établissements dits de banlieue. Autrement dit, ce type de discours dépasse l'urbanité.

En interne, les problèmes entre professeurs et élèves conduisent à une dévalorisation de la professionnalité des enseignants que traduit le cahier de suivi : un document présent dans la salle des professeurs dans lequel chaque enseignant peut mentionner des incidents ponctuels (le manque de papier dans la photocopieuse par exemple). Au moment de l'enquête, il contient les plaintes anonymes des enseignants, à l'égard de leur hiérarchie notamment, certains témoignages allant jusqu'à l'énonciation du suicide (le cahier a pu être consulté, mais non reproduit à la demande de la direction de l'établissement).

La gestion du désordre se réalise principalement par la délégation des enseignants des élèves perturbateurs à la vie scolaire. Une politique sévère des exclusions de cours est alors mise en place et une partie des élèves y répond en cherchant justement à se faire exclure de la classe, dans une sorte de jeu de compétition (du $1^{\text {er }}$ septembre 2014 au 16 janvier 2015, on compte 878 exclusions de cours pour 373 élèves). Dans ce contexte, l'articulation entre ce qui relève du pédagogique et de l'éducatif ne fonctionne plus.

Comme les tentatives des acteurs pour endiguer les phénomènes d'incivilité s'essoufflent, l'arrivée d'animateurs associatifs est perçue telle une opportunité pour recréer une dynamique et revenir à une stabilité.

\subsection{Le lycée 2 : une attention portée à la réussite des élèves}

Dans l'établissement 2, l'opposition au projet, de la part de la cheffe d'établissement, est plus frontale. Dès le début de l'enquête, elle annonce que "l'AFEV n'apporte rien aux élèves » et déclare au chercheur "avoir externalisé le dispositif». Cette assertion se vérifie sur le plan spatial : les animateurs sont relégués dans l'un des premiers bâtiments près de la sortie des élèves, bâtiment qui rassemble le bureau de l'infirmière, du conseiller psychologue et de la MLDS (Mission de Lutte contre le Décrochage Scolaire). Au moment de l'enquête, l'animateur de la MLDS regrette, comme les animateurs de l'AFEV, sa position géographique?.

Dans l'établissement, la division du travail et des espaces scolaires est marquée, si bien que le chercheur, assignée au pôle scolarité, n'a pas pu croiser d'enseignants, preuve que les corps professionnels possèdent leurs territoires spécialisés. Ces spécialisations peuvent être envisagées comme la traduction d'une conception du secondaire que Payet avait décrit pour le collège, c'est-à-dire " comme espace d'apprentissage scolaire achevé " (Payet, 1997, p. 24) au sens où, contrairement à l'élémentaire, pour lequel les enseignants assurent les missions d'apprentissage et de socialisation, ces mêmes missions sont désolidarisées au secondaire. Le refus du partenariat avec les animateurs de l'AFEV trouve son autojustifi-

7. En fin d'année scolaire, un changement de direction a eu lieu. Il a permis de relocaliser l'association dans l'enceinte de l'établissement, ainsi qu'un travail plus étroit entre l'établissement et la MLDS. 
cation dans les performances de l'établissement, que mentionne la cheffe d'établissement.

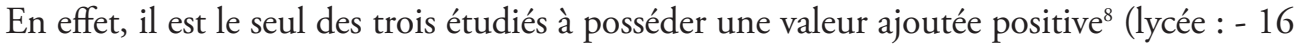
pts ; lycée $2:+1,33$ pts ; lycée $3:-1,33$ pts, MEN, DEPP, 2015). En réalité, les performances doivent davantage être recherchées dans les filières offertes, notamment la filière "petite enfance " qui assure un taux de pression" important, autorisant une sélection scolaire des élèves majoritairement d'origine populaire. Ce n'est pas le cas des deux autres lycées.

\subsection{Le lycée 3 : une culture d'établissement tournée vers le développement général des élèves}

Dans le lycée 3, c'est-à-dire le plus urbain des trois établissements de l'enquête, le projet de partenariat est accueilli sans grand enthousiasme. Le discours du chef d'établissement reste convenu. Il se dit "ravi que la Région propose des actions au sein de l'établissement". Les CPE sont plus loquaces et souhaitent conserver deux prérogatives de leurs missions : d'une part, l'animation de la vie scolaire auprès des élèves ; d'autre part, la conservation de la gestion de l'équipe telle qu'elle est déjà constituée.

Ces deux plans d'intervention font l'objet d'une division du travail. Le premier est incarné par l'un des CPE qui regrette que le poste de l'un de ses deux collègues ait été supprimé et "préférerait sa relocalisation que deux jeunes de l'AFEV». Il décrit son activité davantage dans sa dimension noble, c'est-à-dire plutôt sous l'angle de la transmission de savoirs non institués autour de valeurs telles que la collaboration, la persévérance, la convivialité. Il organise ainsi des activités exceptionnelles comme le cross déguisé avec un concert en fin de journée. Cette façon de souligner sa professionnalité vient recouper l'espace potentiellement occupé par les animateurs de l'AFEV. Le CPE se défie d'ailleurs de l'action de l'association et le credo d'une " jeunesse allant à la rencontre d'une autre jeunesse " ne le convainc guère. Il propose au chercheur de suggérer aux animateurs de l'association la lecture des écrits de Jean-Pierre Terrail "pour agir auprès des élèves faut au moins avoir lu du Terrail" lance-t-il (CPE 1). Il regrette que les animateurs manquent de formation, notamment au regard des catégories sociales et du phénomène du décrochage scolaire, pour agir efficacement auprès des élèves.

Davantage centrée sur le travail intercatégoriel, sa collègue souligne elle aussi la concurrence potentielle que pourraient induire les animateurs de l'association. Elle décrit au chercheur l'attitude des animateurs de l'AFEV lors d'une réunion associant l'ensemble des acteurs de l'établissement et ses partenaires traditionnels (Conseiller d'Orientation Psychologue, conseiller de la MLDS, etc.). Selon elle, les animateurs de l'AFEV auraient

8. La valeur ajoutée "mesure la différence entre les résultats obtenus et les résultats qui étaient attendus, compte tenu des caractéristiques scolaires et sociodémographiques des élèves ", MEN, DEPP, 2015.

9. Il s'agit d'un indicateur qui mesure la difficulté à accéder à une formation, plus il est élevé, plus il est difficile de l'intégrer en raison du plus grand nombre de candidats. 
annoncé être compétents en matière de climat scolaire, d'orientation, de soutien scolaire " en fait en tout " (CPE 2). En bousculant symboliquement des professionnalités stabilisées, les déclarations des animateurs réitèrent la concurrence et contribuent à leur propre isolement. La non-professionnalité des intervenants, qu'ils affichent comme un atout, la mystification de l'autodidacte mis en avant par l'association, jouent en réalité en leur défaveur.

\section{Quelles pratiques en matière de lutte contre le décrochage scolaire?}

La saisie des écarts entre une volonté politique et sa réception auprès des personnels des établissements, selon les contextes dans lesquels exercent les personnels, expliquent en partie les contenus des interventions des animateurs en service civique. Les entretiens réalisés auprès de ces derniers lèvent le voile sur leur rôle objectif : être le relais de l'institution scolaire. L'analyse des entretiens a donné lieu à une typologie de portraits permettant de rendre compte de phénomènes fréquents contribuant à l'exclusion plus qu'à l'intégration des élèves les plus éloignés de la culture légitime de l'école.

Les paragraphes qui suivent exposent ces modalités d'intervention mises en œuvre par les animateurs en service civique selon les lycées et leur contexte, les priorités des CPE où des chefs d'établissement énoncées plus haut.

\subsection{Le lycée 1 : normaliser les élèves}

La situation du lycée 1 conduit à faire exercer aux animateurs un rôle qui consiste à suppléer les CPE et assistants d'éducation. Sur le plan spatial, le bureau des animateurs se situe au cœur du pôle de la scolarité, en cohérence avec la mission qui leur est informellement confiée, tandis que formellement on attend d'eux qu'ils instaurent des relations de convivialité avec l'ensemble des élèves.

En conformité avec les missions informellement attribuées, les animateurs du lycée 1 se définissent en complémentarité des CPE et des assistants d'éducation : "On a une position de médiateur, c'est-à-dire que les CPE, les assistants d'éducation ont beaucoup de travail et souvent [...]. C'est une échappatoire aussi pour eux de nous envoyer un élève qui pose problème (Victor ${ }^{10}$, animateur).

10. Victor, issu de milieu enseignant, a décroché de l'université après deux échecs et a candidaté en vain en tant qu'assistant d'éducation. Ne voulant pas rester inactif en attendant l'année suivante et souhaitant s'engager pour « faire quelque chose d'utile aux autres et qui soit formateur pour moi aussi et qui me rapporte un peu de sous » (ligne 2), il a candidaté à un service civique auprès de l'Afev. Au moment de l'entretien, il a pour projet de postuler à nouveau comme assistant d'éducation ou bien comme animateur l'année prochaine (ligne 4). 
Leurs comportements sont infléchis, d'une part, par la tendance à vouloir que leur travail soit complémentaire à celui des CPE ou des assistants d'éducation, mais aussi à celui des enseignants. Cette double attribution les conduits alors à reproduire, dans le même mouvement, le discours dominant du pôle de la scolarité à l'égard de certains enseignants ("Faut aussi savoir qu'au lycée, y’a des profs qui hésitent pas à virer leurs élèves parce que [...] ils cherchent pas à les comprendre ") et le discours des enseignants vis-à-vis des élèves les plus rétifs : "Ça ne se passe pas parce qu'il n'est pas impliqué. [...]. Après, c'est compliqué, car on a aussi les échos des profs ou des surveillants qui disent: "oui, mais Jim il n'a pas que des problèmes d'école, il touche déjà à la drogue et en gros il arrive, il est déjà défoncé” " (Frédéric ${ }^{11}$ animatrice dans le lycée à propos d'un élève).

Cette double posture est aussi visible dans d'autres arguments qui relèvent plus directement de la pratique pédagogique : "Des fois, leurs profs leur donnent les devoirs au dernier moment dans le cours, donc ils n'ont pas le temps de le noter [...]. Ils ont quelque chose qui s'appelle le Pronote [...] où les profs sont sensés mettre les devoirs [...] les profs les mettent pas forcément. ». Simultanément, ils recommandent aux élèves des interactions de compensation pour "réparer la face " (Goffman, 1973), c'est-à-dire en réhabilitant la valeur sociale positive des enseignants comme condition de la reprise de l'interaction en classe, en recommandant plusieurs stratégies, dont celle qui consiste à s'excuser auprès de l'enseignant désigné comme l'auteur d'une humiliation à leur égard.

En redoublant le discours des CPE, qu'ils acquièrent par imprégnation au pôle de la scolarité, les intervenants contestent l'autorité professorale dans un cadre précis. Ils confortent le CPE, en reconnaissant la nécessité fréquente d'interventions et de façon complémentaire aux mésusages des enseignants (de Pronote par exemple) ; dans le même temps, ils confortent la bonne volonté culturelle de l'élève en l'encourageant à reconnaître la légitimité de l'autorité pédagogique, en rendant visible ses efforts (ne pas réagir, s'excuser quand l'enseignant prononce des paroles blessantes) et par là même, ils légitiment le point de vue des enseignants. S'ils peuvent reproduire le discours, ils n'en partagent pas les contours et leur position reste basse. Ils participent que très rarement au début, puis plus du tout la fin, à la cellule de veille de l'établissement ${ }^{12}$, au service vie scolaire, à la communauté éducative dans l'établissement.

11. Frédérique, issue de milieu enseignant, a obtenu son Master enseignement à l'ESPE, mais a échoué deux fois au concours de professeur des écoles. Suite à ces deux échecs, elle projette, au moment de l'entretien, de se présenter aux mêmes concours dans des académies plus faciles d'accès, comme celles de Versailles et de Créteil. N'ayant pas de diplôme à préparer en attendant les épreuves des concours, elle a souhaité utiliser son temps libre en s'engageant dans le service civique à l'Afev.

12. Il s'agit du Groupe de Prévention du Décrochage Scolaire (GPDS) ou cellule de veille, c'est-à-dire une instance interne qui croise les regards par la réunion de différentes catégories de personnels afin de porter une analyse plus complète et de déboucher sur des réponses plus adaptées. Elle est composée et constituée du chef d'établissement ou de son adjoint, de l'infirmière scolaire, de l'assistante sociale, du COPSY (conseiller d'orientation psychologue), du CPE, et enfin du professeur principal qui souhaite faire part d'un cas particulier. 
Cette division du travail et la mise à la périphérie des intervenants au niveau de l'action expliquent peut-être, en partie, leurs maladresses. Les actions envers les élèves les plus éloignés de l'école, dont rend compte un épisode, parmi d'autres, rapporté par Victor, révèlent la manière dont le dispositif participe aux ruptures scolaires, voire les accélèrent : "La CPE est venue nous voir pour savoir si on pouvait le recevoir [un élève], (...) on est toujours volontaire pour recevoir des élèves (...) je suis allé le voir personnellement pour discuter un peu avec lui (...) l'instant d'après, il s'est enfui par la fenêtre " (...) c'était juste, c'était vers 16 heures et demie, c'était juste avant qu'on parte et euh, donc il se passe ça, nous on peut pas y faire grand-chose et donc on... on s'en va ». Affecté, Victor y voit un conflit de légitimité et réitère ses actions envers le même élève : "On s'en va, je le repère dans la cour et il allait vers le foyer donc, moi, pour qu'ils [les CPE] sachent où leur élève était, je suis allé retrouver la CPE pour... pour le... pour leur dire parce que c'est important, les liens de confiance avec l'équipe éducative aussi".

En s'affirmant du côté de l'ordre scolaire, en le démontrant par la dénonciation, les animateurs accroissent les sanctions des élèves les plus rétifs à l'école.

\subsection{Le lycée 2 : des pratiques socialisatrices à visées correctives}

Les animateurs étant mis à l'écart dans le lycée 2, leurs interventions se situent plutôt dans un registre autour de l'exposition du capital culturel élargie à l'incitation à la mobilité, en favorisant des déplacements en dehors du trajet quotidien école-foyer parental.

Ainsi Maud ${ }^{13}$, titulaire d'un BAFA (Brevet d'aptitude aux fonctions d'animateur) et d'un DUT (Diplôme universitaire de technologie) " carrières sociales ", organise un déplacement collectif en dehors de l'établissement pour découvrir l'école des Beaux-Arts, un musée et un jardin botanique dans la capitale régionale, à $25 \mathrm{~km}$. Les sorties peuvent sembler à but exclusivement ludique, mais les lieux où elles se déroulent nous en apprennent tout autant sur leur contenu, nous y reviendrons : "On fait une sortie par mois [...]. On a fait la chasse aux oufs dans le Jardin des plantes, ça a hyper bien marché, y avait trois parcours, des énigmes, des jeux à faire [...]. On était tous déguisés [...]». La chasse aux œufs se déroule dans le jardin botanique, de distraction, et de conservation d'espèces. Situé dans le centre de l'agglomération, ce lieu est fréquenté par les résidents du quartier qui, en grande partie, habitent des hôtels particuliers.

13. Maud a passé le concours pour devenir assistance sociale, comme sa mère, mais, admissible aux épreuves orales, elle a renoncé pour reprendre une licence de sociologie, après avoir suivi une L1 de psychologie. En attendant l'année suivante, elle s'est dit qu'avec "Beaucoup de temps libre (...), c'est l'occasion de faire mon service civique». De plus, elle voyait dans l'Afev une continuité à la fois dans le domaine et dans les valeurs : "Ça ferait une continuité et ça correspondait totalement à mes valeurs (...) l'ouverture d'esprit et culturelle (...) le fait d'être en équipe, de travailler en équipe ". Son projet à plus long terme reste dans le même domaine au sens large : "Je sais dans quel domaine je veux travailler plus tard: dans le social, et aider les gens en difficultés, les accompagner. » 
Un autre exemple permet de dégager des objectifs socialisateurs : On a fait une autre sortie cinéma, on choisit deux films au xx [cinéma d'arts et essai], un pour les plus grands, un pour les plus petits [...]. On a fait une visite du cinéma et une découverte sur le 7 art [...] ». Le choix du lieu et du film traduit, comme précédemment, une oscillation entre culture populaire supposée et culture mieux valorisée par l'École. En effet, à la différence du multiplex, implanté dans des grandes surfaces commerciales de la périphérie, il s'agit non pas d'aller simplement au cinéma, mais de découvrir et d'apprécier " le $7^{e}$ art » au cœur de la capitale régionale.

Culturellement éloignées de la commune de localisation du lycée et de la plus grande partie de son public, les sorties ne visent pas le simple plaisir ludique. Leur objectif est aussi tacitement socialisateur. Il passe par la reconnaissance d'une hiérarchie des loisirs et de leur légitimité, calquée sur le modèle scolaire. Dans le même temps, les sorties marquent le contraste entre culture populaire rurale et culture urbaine des classes moyenne et favorisée.

\subsection{Le lycée 3 : précipiter les ruptures}

La faible intégration des animateurs dans le collectif établissement s'observe dans les trois lycées, et de manière plus visible dans les lycées 2 et 3 . Contrairement au lycée 1 , où le bureau des animateurs se remplit à la demande des CPE, celui des deux autres lycées est plutôt vide. La capacité des animateurs à mobiliser autour de leur activité est objectivement faible. Alors qu'en entretien, les mêmes animateurs du lycée 3 déclarent avoir une activité intense, dans l'observation, l'ennui n'est jamais loin.

Au lycée 3, la mise à l'écart des animateurs dans le discours des CPE s'accompagne d'une mise à l'écart spatiale, leur bureau ayant été placé au sous-sol, au fond d'un couloir, après le baby-foot.

Écartés du domaine de la transmission culturelle comme de ceux de l'orientation ou du climat scolaire, les animateurs trouvent une possibilité d'autojustifier leurs actions d'intervention grâce à un élève en recherche de stage. Brian, plutôt porté sur "les tutos » (des petits tutoriels filmés portant sur des sujets très divers et postés sur le Web), a d'abord fréquenté l'AFEV pour échanger autour de techniques vidéo, puis a connu un accompagnement serré de la part des animateurs pour sa recherche de stage en entreprise. Brian semble au départ en confiance, puis les exigences de plus en plus scolaires des animateurs liées à son $\mathrm{CV}$, sa lettre de motivation, l'éloignent durablement du dispositif : "ça fait deux semaines qu'on travaille ensemble sur une recherche de stage pour l'année prochaine et cet été (...), mais il donne même pas du sien pour la lettre de motivation, la création de son $C V$ " (Denise ${ }^{14}$,

14. Denise a passé deux années en licence de langues interrompues par deux années à l'étranger, une année sabbatique en Amérique latine et une pour travailler en Martinique. Déçue par les modalités d'enseignement à l'université, elle projette une réorientation et de s'inscrire dans un CAP de chapellerie. Ayant eu « des retards au niveau des inscriptions" (ligne 4), elle "recherchait quoi faire » et «trainait pas mal au local de l'Afev " du fait qu'elle y avait une "très bonne copine qui était là en tant que bénévole " (ligne 2). À plus long terme, elle sou- 
animatrice). Le prétexte des vidéos pour l'attirer et l'amener à un travail scolaire encadré (accepter le contrôle de l'orthographe, recommencer, se relire, faire preuve de réflexivité) a sans doute contribué au décrochage de l'élève certainement pas complètement dupe de la ruse, et dont le parcours par la suite est de plus en plus marqué par l'absentéisme " il a pris une tournure dernièrement et on sait pas trop pourquoi (...) il arrive systématiquement en retard» (Denise). Il dira par la suite en entretien ne plus vouloir fréquenter les animateurs.

La forme de relation entre Brian et les animateurs repose assez visiblement sur la mission que se donnent ces derniers : scolariser Brian. Ici l'enjeu est fort pour les acteurs associatifs. Il s'agit en effet pour eux d'être reconnus comme participant de la réussite scolaire des élèves de l'établissement. Cette reconnaissance dépend du succès de l'action ; c'est pourquoi Brian doit réussir. Au moment de la prise en charge de l'élève par les membres de l'AFEV, l'ensemble de la communauté éducative disait ne pas savoir "quoi en faire". Réussir avec Brian visait explicitement la reconnaissance d'une faculté du dispositif un moment entrevue. Il s'agissait, par le biais de la réussite de l'action, de montrer les limites de l'expertise pour en revenir aux catégories indigènes des "qualités humaines et authentiques" (Antoine ${ }^{15}$, animateur proche de Brian). L'échec de l'action a non seulement précipité la rupture de Brian d'avec l'école, mais s'est accompagné d'une description très personnalisée de l'élève de la part des animateurs.

\section{Conclusion}

L’originalité du propos est de montrer en quoi chacune des étapes de la mise en place du dispositif contribue à l'échec des objectifs annoncés.

La mobilisation d'une méthodologie d'enquête ethnographique a permis d'étudier à un degré fin de granularité des logiques d'acteurs à trois niveaux (celui des concepteurs, celui des acteurs traditionnels de l'école, et de néo-intervenants en milieu scolaire dans trois lycées professionnels). Le couplage de la théorie de la négociation et de l'analyse du discours a permis de formuler des indices relatifs à la co-constrution de normes, de valeurs à la genèse du dispositif, et de rendre compte des discours produits selon les statuts des individus dans des contextes sociaux variés.

\footnotetext{
haite rester en lien avec le secteur associatif mais dans un autre domaine, plus proche des milieux artistiques. 15. Antoine, issu de milieu cadre supérieur dans le secteur privé, par ses parents, a enchaîné un BTS «dans le social», dont il dira plus loin qu'il s'agit d'un BTS «SP3S, Secteur et prestations des services sanitaires et sociaux" et cinq stages diversifiés du point de vue des employeurs, mais aussi sur le plan géographique : "J'ai fait des stages, beaucoup, à Sarguemines, c'est dans le 57 en Moselle, en mission locale avec une conseillère en insertion, professionnelle et dans le logement et j'ai fait un stage avec des éducateurs de rue toujours dans le 57, et voilà, après, jai eu des expériences avec les maisons de retraite, avec les restos du cour, et la croix rouge. "
} 
Les analyses montrent que sans la construction d'accords explicites dans la structuration et le fonctionnement d'un collectif pour la mise en place de tels dispositifs, les principaux bénéficiaires perdent.

C'est le cas de la Région. L'absence de dialogue avec les acteurs des établissements ne lui permet pas d'envisager la complexité des différences inter-établissement, et ainsi d'élaborer des solutions au cas par cas selon des besoins identifiés.

Les établissements perdent puisqu'ils sont contraints d'accueillir un dispositif imposé et de s'en accommoder. Ils tentent alors de faire des animateurs, dans le meilleur des cas, des suppléants de l'ordre scolaire, ou à défaut de s'en défaire.

De tels éléments de départ créaient des contextes d'action défavorables aux animateurs. Arrivés sur le terrain dans le cadre d'une politique d'insertion, ils sont au quotidien très souvent livrés à eux-mêmes au sein des établissements. Les injonctions contradictoires, entre l'encouragement de la Région et de l'association conjuguées avec leur subordination ou leur mise à l'écart des équipes éducatives, les conduisent à osciller entre deux tendances : scolariser des formes de convivialité (les loisirs, les discussions informelles, voire la distribution de chocolats ne sont jamais sans but) pour normaliser les élèves, et en cas d'échec, précipiter les ruptures scolaires. De façon déclarative, deux élèves dans ce cas ont été identifiés par les équipes éducatives : Jim et Brian, dans deux établissements distincts.

L'enquête montre ainsi que les formes de conflictualité, voire les idéologies éducatives ou d'emploi qui s'actualisent dans le dispositif conduisent à produire les effets adverses à ceux affichés.

Nos conclusions sont certes fondées sur des observations intensives et des entretiens menés dans trois lycées professionnels à l'occasion d'une expérimentation. En conséquence le propos ne concerne qu'un nombre de cas limités. Elles nous semblent néanmoins valoir au-delà de ces établissements. Les travaux de Cadet (Cadet et al., 2000) mais aussi ceux Guitton (2000), ou encore Champy-Remoussenard (2004) pour n'en citer que quelquesuns sur le dispositif "emplois-jeunes " possèdent des points de convergences avec les nôtres, et invitent à des comparaisons plus systématiques d'une part et massives d'autre part en vue de mieux cerner de tels effets. 


\section{Bibiographie}

Armand A., Bisson-Vaivre C. \& Lhermet P. (coord.) (2013), Agir contre le décrochage scolaire : alliance éducative et approche pédagogique repensée, Rapport Inspection générale de l'Éducation nationale \& Inspection générale de l'administration de l'Éducation nationale et de la Recherche.

Attarça M. \& Chomienne H. (2013), «Les chefs d'établissements publics scolaires français face aux enjeux de la nouvelle gestion du système éducatif ", @GRH, 9, (4), pp. 35-66.

Barrère A. (2013), "Les établissements scolaires à l'heure des dispositifs ", Carrefours de l'éducation, 361, vol .2/361, pp. 9-13.

Ben Ayed C. (2009). Le nouvel ordre éducatif local. Mixité, disparités, luttes locales, Paris, PUF.

Bernard P.-Y. (2011), Le décrochage scolaire, Paris, PUF.

Bezès P. (2009), Réinventer l'État. Les réformes de l'administration française (1962-2008), Paris, PUF.

Blaya C. (2013). "Le décrochage scolaire dans les pays de l'OCDE », Regards croisés sur l'économie, 2, pp. 69-80.

Bourdieu P. (1978), «La jeunesse n’est qu’un mot. Entretien avec Anne-Marie Métailié », paru dans Les jeunes et le premier emploi, Paris, Association des Ages, pp. 520-530. Repris in Questions de sociologie, Paris, Éditions de Minuit, Ed. 1992, pp. 143-154.

Boutet J. \& Maingueneau D. (2005), « Sociolinguistique et analyse de discours : façons de dire, façons de faire ", Langage et société, 114, (4), pp. 15-47.

Bruno F., Félix C., Saujeat F. (2017), «L'évolution des approches du décrochage scolaire », Carrefours de l'éducation, 43(1), pp. 246-271.

Bruno F., Méard J. \& Walter E. (2013), «Les dispositifs français de lutte contre le décrochage scolaire en collège : ce qui est prescrit et ce qui est mis en œuvre ", L'Orientation scolaire et professionnelle, 42(4), pp. 439-459.

Bourquelot L. \& Derouet J.-L. (1994), "Les territoires désajustés ", in Charlot B. (dir), L'École et le Territoire, Paris, Armand Colin.

Broccolichi S., Ben Ayed C., Mathey-Pierre C. \& Trancart D. (2007), «Fragmentations territoriales et inégalités scolaires : des relations complexes entre la distribution spatiale, les conditions de scolarisation et la réussite des élèves ", in Mesurer les inégalités sociales de scolarisation: méthodes et résultats, Éducation et formations, ministère de l'Éducation nationale, pp. 31-48. 
Cadet J.-P., Diederichs-Diop L., Fournié D. \& Guitton C. (2000), « Aide-éducateur : quel avenir pour la fonction, quel devenir pour les jeunes? ", Documents Céreq, série "Évaluation ", $\mathrm{n}^{\circ} 151$, septembre.

Carion F. (2010), " La communication associative ", Communication, Vol. 28/1, pp. 193-206.

Chamboredon J.-C. (1966), "La société française et sa jeunesse ", in Darras (dir.), Le Partage des bénéfices. Expansion et inégalité en France. Préface de Gruson C., Paris, Éditions de Minuit, "Le sens commun ».

Champy-Remoussenard P. (2004), «Emplois-jeunes : les compétences «relationnelles» en question ", in Champy-Remoussenard P. et Meyer V. (sous la direction de), Etre emploijeune et après? ? Presses universitaires de Nancy, pp. 145-161.

Commission européenne, Direction générale de l'Éducation et de la Culture (2005), Study on access to education and training, basic skills and early school leavers. Lot 3:Early school leavers, final report.

Dutercq Y., Gather Thurler M. \& Pelletier G. (Dir) (2015), Le leadership éducatif : Entre défi et fiction, Louvain-la-Neuve, Belgique, De Boeck Supérieur.

Frandji D., Demeuse M., Greger D. \& Rochex J.-Y. (2009), Comparaison des politiques d'éducation prioritaire en Europe, Rapport de recherche pour la Commission européenne, programme Socrates 2, Lyon, INRP.

Guitton C. (2000), " La professionnalisation, nouvelle catégorie de l'intervention publique », Formation Emploi, n 70 , septembre.

Guiliani F., Payet J.-P (2014), «Introduction. Les logiques scolaires de la proximité aux familles ", Éducation et Sociétés, 5-141, pp. 74-85.

Goffman E. (1973), La mise en scène de la vie quotidienne 1, La présentation de soi / Erving Goffman, trad. de l'anglais par Alain Accardo, Paris, Éditions de minuit (1992), cop. 1973.

Hood C. (1991), “A Public Management for All Seasons?”, Public administration, 69, pp. 3-19.

Kakpo S., Netter J. (2013), «L'aide aux devoirs. Dispositif de lutte contre l'échec scolaire ou caisse de résonnance des difficultés non résolues dans la classe ", Revue française de pédagogie, 182, pp. 55-70.

Kotler P. \& Zaltman G. (1971), "Social marketing: An approach to planned social change", Journal of Marketing, 35, pp. 3-12.

Minassian L. (2017), "Un exemple d'alliance éducative en lycée professionnel : un effet incertain sur le climat scolaire ", in Ferréol G. (dir.), Climat scolaire et décrochage, Bruxelles, E.M.E. Intercommunication. 
Millet M., Thin D. (2017), "Ni gêneurs, ni partenaires, mais sous pression institutionnelle. Les familles d'élèves en ruptures scolaires ", Administration et Éducation, 153(3), pp. 81-85.

Millet M \& Thin D. (2005), Ruptures scolaires. L'école à l'épreuve de la question sociale, Paris, Presses universitaires de France, série «Le lien social ».

Payet J.-P. (1997), "Le 'sale boulot' " : Division morale du travail dans un collège en banlieue. Les Annales de la recherche urbaine ", L'école dans la ville, 75, pp. 19-31.

Pirone F. \& Rayou P. (2012), « Nouveaux internes, anciens décrocheurs : de l'évolution de la forme scolaire ", Revue française de pédagogie, 179, pp. 49-62.

Pollitt C. \& Bouckaert G. (2011), Public Management Reform: A Comparative Analysis New Public Management, Governance, and the Neo-Weberian State, Oxford: University Press.

Pont B., Nusche D. \& Hopkins D. (2008), Améliorer la direction des établissements scolaires, Rapport OCDE.

Rey O. (2013), "Décentralisation et politiques éducatives ", Dossier d'actualité Veille et Analyses IFÉ, $\mathrm{n}^{\circ}$ 83, avril, Lyon, ENS Lyon.

Schmoll P. (1996), "Production et interprétation du sens : la notion de contexte est-elle opératoire?", Scolia, 6, pp. 35-255.

Strauss A. (textes réunis par Isabelle Baszanger) (1992), La Trame de la négociation. Sociologie qualitative et interactionnisme, Paris, L'Harmattan.

Strauss A. (1978), Negotiations. Varieties, contexts, processes and social order. San Francisco, Jossey-Bass publishers.

Wieser F. (1901), Über die gesellschaftlichen Gewalten [Des pouvoirs sociaux], Prague, Presses Universitaires Carl Ferdinand.

Weber M. (2013), La domination, Paris, La Découverte, coll. "Politique \& sociétés ", (édition augmentée 1914). 